\section{LA TRANSFERENCIA TECNOLÓGICA COMO PRÁCTICA DE RESPONSABILIDAD SOCIAL UNIVERSITARIA}

\author{
Gloria Naranjo Africano \\ Universidad del Norte \\ ORCID iD: https://orcid.org/0000-0001-9610-1604 \\ gnaranjo@uninorte.edu.co
}

Camilo Mejía Reatiga Universidad del Norte ORCID iD: https://orcid.org/0000-0002-8687-2250 cmejia@uinorte.edu.co

Cómo citar este artículo/Citation: Naranjo Africano, G., Mejía Reatiga, C. (2018). La transferencia tecnológica como práctica de responsabilidad social universitaria. Arbor, 194 (789): a472. https://doi.org/10.3989/arbor.2018.789n3014

Recibido: 06 septiembre 2017. Aceptado: 05 febrero 2018.

RESUMEN: La responsabilidad social universitaria (RSU) constituye un desafío para las universidades y plantea el reto de alcanzar estándares que involucren y beneficien a todas las partes interesadas: a los académicos por medio del estudio de los elementos en orden a su adecuado funcionamiento, $y$ al personal de administración y servicios a través del diseño de políticas y estrategias para el fomento y la regulación de la RSU. Aunque la bibliografía relaciona de manera general los procesos de transferencia tecnológica (TT) y la RSU, no se ha profundizado en sus especificidades e implicaciones. Este trabajo tiene como propósito, a partir de una revisión de la bibliografía, definir cómo se puede entender la TT como práctica de RSU, sobre la base de una serie de propuestas de actividades concretas y de las posibilidades de investigación que el tema plantea.

PALABRAS CLAVE: Responsabilidad social universitaria; transferencia tecnológica; capital social; apropiación social.

\section{TECHNOLOGICAL TRANSFER AS A PRACTICE OF UNIVERSITY SOCIAL RESPONSIBILITY}

Copyright: (C) 2018 CSIC. Este es un artículo de acceso abierto distribuido bajo los términos de la licencia de uso y distribución Creative Commons Reconocimiento 4.0 Internacional (CC BY 4.0).

ABSTRACT: University Social Responsibility (USR) is a challenge today for universities that must aim to meet standards that involve and benefit all stakeholders; for academics as an object of study of the elements required for their adequate performance and for practitioners in the design of policies and strategies for the development and regulation of USR. Although the literature relates the general processes of Technological Transfer (TT) to the USR, its specificities and implications have not been explored in depth. This paper aims, from a literature review, to identify how and under what actions the TT can be understood as an USR practice; based on the establishment of specific proposals and actions and on possible research opportunities for this subject.

KEYWORDS: University Social Responsibility; Technology Transfer; social capital; social appropriation. 


\section{INTRODUCCIÓN}

A partir de los años 80, un entorno determinado por la denominada sociedad del conocimiento, el incremento de la importancia de las competencias científicas y tecnológicas y los cambios en las políticas de financiación gubernamental significaron una renovación del contrato social de la universidad, en el que las universidades debían dar respuesta a las necesidades de los "usuarios" en la economía y en la sociedad (Martin, 2003). Esto se relaciona con una transformación en la misión tradicional de docencia e investigación de la universidad y conlleva un mayor acercamiento de estas instituciones a las demandas y a las necesidades de diferentes actores sociales, lo que la bibliografía denomina como una tercera misión (Etzkowitz y Leydesdorff, 2000, p. 110). Esto ha llevado a la universidad a convertirse en un actor clave en el desarrollo social y económico de su región de influencia, a través de una vinculación mucho más estrecha con los diferentes agentes de su entorno (Vega Jurado, Manjarrés Henríquez, Castro Martínez y Fernández de Lucio, 2011; Wang, Hu, Li y Pan, 2016), y ha permitido mejorar la competitividad y la creación de riqueza en las regiones donde se lleva a cabo esta dinámica (Barnes, Pashby y Gibbons, 2002. p, 396).

Las principales actividades asociadas a esta tercera misión se han enmarcado en procesos de transferencia tecnológica que para el caso de universidades en países desarrollados se traducen en la generación de patentes, licencias, empresas spin-off e innovaciones, así como en la realización de consultorías especializadas del trabajo articulado con empresas y en el sustento a la creación de estructuras tales como las oficinas de transferencia tecnológica (Siegel, Waldman, Atwater y Link, 2003). Particularmente en Latinoamérica se ha adoptado una perspectiva según la cual este proceso denominado extensión universitaria se asocia a la función social de la universidad, a la proyección de la cultura universitaria y a la preocupación por los problemas nacionales (Tünnermann Bernheim, 2010. p, 119). Sin embargo, a partir de la década de 1990, se materializa en América Latina un cambio en las políticas de ciencia y tecnología que, inspiradas en la experiencia de los países desarrollados, promueve en las universidades la adopción de la denominada tercera misión (Vega Jurado et al., 2011, p. 74) desde una perspectiva tecnológica.

Es así como los procesos de transferencia tecnológica cobran relevancia en el interior de las universidades como indicadores de las relaciones con su entorno, por lo que las universidades realizan continuamente esfuerzos para continuar sus vínculos colaborativos, sobre todo con el sector empresarial. Sin embargo, se está viviendo un momento clave en la reinvención conceptual de la misión social de las universidades -aquella de cara a la comunidad, al impacto en su entorno- apoyado en los cambios que conducen hacia la transferencia de conocimiento, intercambio y coproducción de conocimiento, migrando hacia un concepto de universidad comprometida (Conway, Humphery, Benneworth, Charles y Younger, 2009).

De manera particular, la relación entre la universidad y el entorno, así como su papel en la difusión del conocimiento como respuesta a problemas sociales, se sustentan en la hoy denominada responsabilidad social universitaria (RSU), que evidencia el compromiso de la universidad con las instituciones y actores con las que interactúa sobre la base de un sistema de valores que tiene en cuenta los derechos humanos y el desarrollo sostenible. En la bibliografía se han desarrollado elementos teóricos que hoy configuran las nociones básicas de la responsabilidad social en el interior de la universidad (Gaete Quezada, 2015; GascaPliego y Olvera-García, 2011; Siracusa, Ardiles y Pulido, 2012; Vallaeys, 2006), centrados principalmente en acciones de intervención social y de relación directa con la comunidad interna y externa de la universidad. Sin embargo, aún se reconoce la necesidad de desarrollar y hacer mucho más operativo el concepto de responsabilidad social universitaria, de tal forma que se fortalezca el corpus de bibliografía que apoya este trabajo en el interior de las universidades.

Teniendo en cuenta la relación existente entre la denominada tercera misión, que hoy se apoya en gran parte en procesos de transferencia tecnológica, y lo que define la responsabilidad social universitaria, este trabajo tiene como propósito identificar puntos comunes de actuación que desde el ejercicio de los procesos de transferencia tecnológica puedan entenderse también como prácticas de responsabilidad social universitaria, colocándose en línea con el enfoque de universidad comprometida. Por ello busca responder al interrogante sobre cómo la transferencia tecnológica puede constituir una práctica de responsabilidad social universitaria, tomando como base una revisión sistemática de la bibliografía sobre responsabilidad social universitaria y del estado de la cuestión sobre transferencia tecnológica, identificando cómo y bajo qué acciones la transferencia tecnológica puede ser entendida como una práctica de responsabilidad social universitaria, y planteando oportunidades de investigación futura sobre esta temática. 
Las conclusiones indican que los procesos de transferencia tecnológica evidencian el compromiso social de la universidad, considerando aspectos como: (i) la transferencia tecnológica incorpora problemas sociales que conllevan la generación de transformaciones, (ii) el concepto de conocimiento socialmente robusto, (iii) la divulgación de los procesos de transferencia tecnológica como mecanismo de apropiación social del conocimiento y (iv) el fortalecimiento del vínculo entre las distintas partes interesadas como parte del capital social de los procesos de transferencia tecnológica.

Este artículo se dividirá en las siguientes secciones (i) el marco teórico sobre responsabilidad social universitaria, (ii) la transferencia tecnológica en la universidad, (iii) la responsabilidad social de la transferencia tecnológica, (iv) la transferencia tecnológica como práctica de responsabilidad social universitaria y (v) conclusiones.

\section{LA RESPONSABILIDAD SOCIAL EN LA UNIVERSIDAD}

En las universidades ha aumentado la necesidad de demostrar que efectivamente su funcionamiento $y$ razón de ser se orientan a la satisfacción de las necesidades y problemas de las personas, grupos o instituciones con los que interactúan (Gaete, 2011, p. 110). Es así como la responsabilidad social universitaria es concebida como un proceso global que trasciende los procesos o funciones misionales de docencia, investigación y extensión, llevando a cabo de manera sinérgica e integral el compromiso de la colectividad universitaria hacia la vinculación social con las comunidades, donde particularmente se debe conformar un sistema de valores éticos fundamentado en los derechos humanos y en el desarrollo sostenible (Aristimuño y Rodriguez Monroy, 2014, p. 377).

La evolución del concepto de responsabilidad social universitaria ha presentado cuatro etapas diferenciadas temporal y circunstancialmente: (i) el paso de una universidad de élite a una universidad inclusiva, generando oportunidades para más personas, independientemente de su posición social y nivel económico, (ii) la aparición de una universidad con proyección social, estableciendo mayor contacto con las comunidades nacionales e internacionales y generando beneficios para ambas partes, (iii) la aparición de una universidad comprometida con el medio ambiente, a partir de la Cumbre de la Tierra, realizada en Brasil en 1992, en la que el concepto de desarrollo sostenible alcanza al sector universitario, quedando integrado en su compromiso con la sociedad (Araque Acevedo, Rubio Guerrero y Uribe Macías, 2015) y (iv) la aparición de una universidad socialmente responsable, el uso de la expresión responsabilidad social universitaria y el inicio del debate acerca de responsabilidad social, la autonomía y la libertad académica en las universidades (Cardona Zuleta, 2010).

La investigación sobre la responsabilidad social en las universidades emergió desde finales de los 90 sustentada en gran parte en lo expuesto en la Conferencia Mundial de Educación Superior de las Naciones Unidas para la Educación, la Ciencia y la Cultura, donde se reconoció que la responsabilidad de las universidades no es solo educar a los jóvenes para su futuro profesional sino también para que sean capaces de aplicar sus habilidades y talentos con el mayor sentido social posible (Conferencia Mundial sobre la Educación Superior, octubre 1998). Esto fue ratificado en el año 2009, reconociendo además la necesidad de que estas instituciones de educación contribuyan a la formación de ciudadanos dotados de altos principios éticos (Conferencia Mundial sobre la Educación Superior, julio 2009).

Al compromiso en la formación de los estudiantes se suma la relación de las universidades con sus distintas partes interesadas, con las personas y organizaciones sobre las que influyen, así como con aquellos grupos que pueden incidir sobre ellas, de manera negativa o positiva (Aristimuño y Rodriguez Monroy, 2014, p. 377). Dentro de las Ilamadas partes interesadas, Vallaeys, Cruz y Sasia (2009) hacen referencia a una amplia gama de individuos, tales como: el personal no docente, los docentes y el personal de investigación, las autoridades, los estudiantes, los proveedores, los graduados, los reclutadores, los competidores, las comunidades locales, las organizaciones asociadas y las entidades públicas gubernamentales. Por ello, en el marco de lo expuesto, la responsabilidad social universitaria requiere ser apreciada desde tres niveles: (i) responsabilidad social universitaria interna, que incorpora a los estudiantes, docentes, investigadores, directivos y personal administrativo, (ii) responsabilidad social universitaria externa, que incluye empleadores, graduados, proveedores y socios estratégicos directos y (iii) responsabilidad social universitaria hacia el estado, la sociedad, el desarrollo y el medio ambiente global (Gasca-Pliego y Olvera-García, 2011, p. 50).

\subsection{Condiciones de la responsabilidad social universitaria}

La responsabilidad social universitaria se apoya en una serie de elementos que hacen evidente su existencia en el interior de las universidades, muchos de ellos intrínsecos a su quehacer. Es necesario estable- 
cerlos, reconocerlos y hacerlos explícitos para el desarrollo de esta función. Boyle, (2007) reconoce en su trabajo algunos de estos elementos:

1. Valores morales y cívicos y la misión de la institución educativa: inspiran e influyen en el entorno educativo con respecto al compromiso con la comunidad.

2. Currículo oculto: lecciones no declaradas que se aprenden dentro y fuera del aula.

3. Ambiente educacional: creación de un entorno para el aprendizaje efectivo, especialmente influyente en lo que respecta a la educación moral.

4. Vecindad y ciudadanía: en la medida en que las universidades se comportan como buenos vecinos, se convierten en un actor clave en su entorno de influencia, demostrando la "ciudadanía" de la institución.

5. Comunicación: la comunicación de la universidad con su entorno permite responder a las demandas de los grupos de interés, por lo que se ha de realizar una rendición de cuentas y lograr la participación de la comunidad (Garde Sánchez, Rodríguez Bolívar y López-Hernández, 2013, p. 737).

\subsection{Prácticas de responsabilidad social universitaria}

Entre las prácticas reconocidas e identificadas en la bibliografía en las cuales se apoya una universidad para hacer explicita su responsabilidad social, están:

i. La docencia: en el marco de la responsabilidad social universitaria, la docencia es una función dirigida a la formación de profesionales. El docente no solo contribuye con la formación de profesionales cualificados sino que genera productos tangibles con importancia social y científica al articularla con la investigación y la extensión (Guadrón, Silva, Acosta y Piñero, 2012, p. 271).

ii. La gestión ambiental: se ha concebido como una respuesta social desde las universidades la inclusión de los temas ambientales en sus actividades de enseñanza, investigación, extensión a la comunidad y gestión de la universidad (Chiappetta Jabbour, 2010, p. 52).

iii. La educación ética: Larrán Jorge y Andrades Peña (2014, p. 150) encontraron que particularmente para el caso de las universidades españolas la formación ética responde a lo exigido por el proceso de Bolonia. Esta es una práctica que cada vez más se está incorporando en las universidades, asociada a los problemas de discernimiento ético que se han manifestado en la población egresada, lo cual ha promovido más esta formación.

iv. La extensión: su finalidad es establecer un nivel de comunicación y de acción entre la universidad y la comunidad, a través de procesos intra y extrauniversitarios mediados por la praxis (Torres Pernalete y Trápaga Ortega, 2010, citado en Gasca-Pliego y Olvera-García, 2011, p. 50); está asociada a la capacidad de la universidad de atender a necesidades y problemáticas sociales.

v. El servicio-aprendizaje: constituye el método por el cual los estudiantes pueden adquirir valores morales y cívicos, conocimiento académico, y desarrollar habilidades en el mundo real (Boyle, 2007, p. 87).

vi. Los servicios de infraestructura: son todo tipo de instalaciones que una universidad, aparte de sus funciones de investigación y enseñanza, ofrece para sus grupos de interés (Esfijani, Hussain y Chang, 2013, p. 278).

\subsection{Impactos de la responsabilidad social universitaria}

Vallaeys y Carrizo (2006) reconocen que la formación humana y profesional y la construcción de nuevos conocimientos constituyen las principales actividades de la universidad, y que por consiguiente tienen impactos específicos distintos a los generados por las empresas. De manera integrada, Vallaeys et al. (2009) señalan que la responsabilidad social universitaria tiene cuatro categorías de impactos:

i. Impactos de organización (laboral y ambiental): la universidad genera impactos en la vida de quienes tienen algún tipo de vínculo o relación con ella. Así mismo también debe incorporar estrategias que aseguren el control y el adecuado manejo de la contaminación en su medioambiente (desechos, deforestación, polución atmosférica por transporte vehicular, entre otras), de tal forma que no genere impactos negativos en su entorno de influencia.

ii. Impactos sociales (extensión, transferencia y proyección social): la universidad tiene un impacto sobre la sociedad y sobre su desarrollo económico, social y político; es un referente y un actor social que puede promover o no el progreso, que puede crear o no capital social, que puede vincular o no la educación de los estudiantes con la realidad social exterior. 
iii. Impactos educativos (formación académica): la universidad tiene un impacto directo sobre la formación de los jóvenes y de los profesionales, sobre su manera de entender e interpretar el mundo, de comportarse en él y de valorar así su vida.

iv. Impactos cognitivos (investigación y epistemología): la universidad orienta la producción del saber y de las tecnologías, influye en la definición de lo que se llama socialmente verdad, ciencia, racionalidad, legitimidad, utilidad, enseñanza.

La articulación de las necesidades de las partes interesadas y de los impactos de la institución universitaria conducen a establecer y a definir los cuatro ejes de responsabilidad social universitaria (Vallaeys et al., 2009), que se apoyan en la gestión socialmente responsable de:

i. La participación social: la participación de la universidad en la comunidad. Se trata de la reunión de diversos actores universitarios y no universitarios alrededor de un proyecto social consensuado, de tal forma que se asegure un aprendizaje permanente colectivo y se contribuya al mismo tiempo a la solución de problemas sociales concretos.

ii. La formación profesional y ciudadana: la formación académica en su temática, organización curricular, metodología y propuesta didáctica. Una buena formación profesional y ciudadana implica que exista una estrecha vinculación entre la universidad y las necesidades reales de la sociedad.

iii. La gestión social del conocimiento: la producción y difusión del saber, la investigación y los modelos epistemológicos promovidos desde el aula. En este eje se ubica lo concerniente a la responsabilidad social de la transferencia tecnológica por parte de la universidad.

iv. El campus responsable: implica la gestión socialmente responsable de la organización y de sus procedimientos institucionales, del clima laboral, del manejo del talento humano, de los procesos democráticos internos y del cuidado del medio ambiente, así como de la relación de la universidad con proveedores, vecinos y comunidad en general.

\section{LA TRANSFERENCIA TECNOLÓGICA DESDE LA UNIVERSIDAD}

Un cambio en el contrato social de la universidad y el estado que implicó una contribución de los resultados de la investigación a las demandas sociales y económicas (Martin, 2003) y a los procesos de generación del saber, más conectado con los escenarios transversales de carácter social y económico bajo el concepto de modo 2 de producción de conocimiento (Gibbons, Limoges, Nowotny, Schwartzman, Scott y Trow, 1994) y particularmente un enfoque económico de estudio de esta vinculación de la universidad con su entorno socioeconómico es la triple hélice de la interacción universidad-industria-gobierno (Leydesdorff y Etzkowitz 1996, p. 279). Estos elementos constituyeron el contexto en el que, además de las misiones tradicionales de docencia e investigación de la universidad, se incorporara lo que en la bibliografía se conoce como tercera misión, también denominada función de desarrollo económico y social, servicios a la comunidad o extensión (Beraza Garmendia y Rodríguez Castellanos, 2007, p. 50), para dar respuesta a las demandas de comunidades cercanas a la universidad, tanto del sector público como del privado.

Esta tercera misión ha implicado considerar la universidad como una institución de educación para toda la vida y de prestación de servicios científicos mediante la transferencia de tecnología al sector productivo con el objetivo último de ayudar a la consecución de una ventaja competitiva a nivel nacional o regional. En muchos países la universidad se ve como un importante elemento en el desarrollo regional (Beraza Garmendia y Rodríguez Castellanos, 2007, p. 50).

De manera específica existen tres enfoques conceptuales para la definición de la tercera misión: (a) desarrollo de un conjunto de actividades de cooperación de la universidad con agentes públicos y privados para transferir a la sociedad conocimiento y tecnología para la innovación y el bienestar social; (b) compromiso social y comunitario en la solución de los problemas del entorno o del territorio en el cual se desarrolla; este enfoque es similar al que se ha venido consolidando en América Latina en el que la universidad asume una "misión social" capaz de vincularla más estrechamente con la sociedad y sus problemas (Tünnermann Bernheim, 2010); y (c) surgimiento de la universidad empresarial o emprendedora, que conduce no solo a la creación de empresas por parte de estudiantes, graduados y profesores sino también al desarrollo de actividades de transferencia de tecnología a la empresa, lo que incluye la comercialización de la producción científica y la interacción universidad-gobierno-industria para el fortalecimiento de los sistemas nacionales de innovación en el modelo de triple hélice (Bueno Campos y Casani Fernández de Navarrete, 2007, citado en Vera Salazar, Galvis-Lista y González Zabala, 2013, p. 136). 
Un hecho que incidió en las relaciones de la universidad con agentes de su entorno fue la denominada Ley Bayh-Dole, aprobada en 1980 en un esfuerzo por acelerar la difusión y comercialización de nuevos conocimientos producidos en las universidades (Hayter, 2016), a lo que se sumaron diversas fuerzas que promovieron cambios significativos en las políticas de patentes universitarias, que se manifestaron en la decisión de muchas universidades de establecer oficinas de transferencia de tecnología (Roessner, Bond, Okubo y Planting, 2013) para que luego surgieran en el entorno universitario una variedad de instituciones especializadas en la comercialización de tecnología a través de una spin-off o de mecanismos financieros de apoyo a este tipo de iniciativas, produciéndose un cambio de actitud en los órganos de dirección de las universidades y entre sus miembros hacia la creación de este tipo de empresas (Beraza Garmendia y Rodríguez Castellanos, 2007, p. 35). Es así como gran parte de las relaciones de la universidad con su entorno se establecieron principalmente con empresas, apoyadas en mecanismos de vinculación propios de procesos de transferencia tecnológica tales como patentes, licencias, empresas spin-off y contratos de consultoría especializada.

D’Este y Perkmann (2011, p. 318) reconocen que la creciente participación de las universidades en la transferencia de tecnología y en la comercialización ha generado nuevos retos acerca de su naturaleza y misión; desde la perspectiva del modelo de la triple hélice este representa la disposición de profesores universitarios y graduados a participar activamente en la transferencia de tecnología (Etzkowitz y Leydesdorff, 2000, p. 110).

En cuanto a los canales o mecanismos de transferencia tecnológica, Merchán Hernández (2012, p. 197) ha agrupado las formas de cooperación más generales entre la universidad y la empresa en: (i) proyectos de I+D y asesoramiento tecnológico, (ii) formación o intercambio de personal, (iii) participación en empresas spin-off, (iv) explotación de resultados de propiedad intelectual y (v) utilización de instalaciones o materiales de la universidad. Se reconoce también la existencia de mecanismos formales e informales de transferencia de tecnología (Okamuro y Nishimura, 2009, p. 275) de acuerdo a los costes generados, de tal forma que los menos costosos y más usados son la consultoría, la utilización de los equipos técnicos de la universidad por parte de las empresas y la implementación de procesos de transferencia de conocimientos a la empresa, mediante diferentes alternativas como la vinculación de graduados universitarios, la formación en procesos especializados o el suministro de información (Fromhold-Eisebith y Werker, 2013, p. 626). La implementación de todos estos mecanismos está orientada a promover una alta calidad de la investigación, de la innovación y del progreso económico y social (Guerrero, Urbano, Cunnigham y Organ, 2014, p. 427).

Cabe mencionar que los procesos de transferencia tecnológica se ven permeados por las características de la universidad, tales como su naturaleza pública o privada, su calidad académica y el tamaño y antigüedad de la oficina de transferencia tecnológica, así como también por las condiciones locales de la demanda de alta tecnología y por los términos del contrato de licencia (Siegel, Veugelers y Wright, 2007, p. 656). A ello se suman otros factores como la conexión social, la confianza, las políticas de propiedad intelectual, la capacidad tecnológica (Bierly III, Damanpour y Santoro, 2009, p. 498), la valoración de la investigación, y la participación de estudiantes, docentes y grupos de investigación (Closs, Ferreira, Soria, Sampaio y Perin, 2012, p. 104).

El contexto donde se lleva a cabo la transferencia juega también un papel importante. Con relación a esto Casper (2013, p. 1314), por ejemplo, mantiene que la calidad del entorno regional de una universidad puede impactar significativamente su éxito en la comercialización de la ciencia. La proximidad de los sectores estratégicos, la construcción de comunidades, el establecimiento de modelos participativos de co-creación entre los usuarios y las comunidades de desarrollo en un modelo de innovación abierta (Freitas, Mayer, Arnab y Marshall, 2014) favorecen los procesos de transferencia y destacan la importancia de la interacción con los actores sociales en el marco este tipo de procesos.

\section{METODOLOGÍA}

La revisión de los aspectos asociados con la responsabilidad social universitaria y la transferencia tecnológica muestra varias diferencias entre ambas, de lo que se podría presumir que estos términos no tienen factores de convergencia; es así como a partir de una revisión de la bibliografía se pretendió identificar elementos que permitieron interconectar ambos conceptos y encontrar en la transferencia de tecnología una forma de hacer operativa la responsabilidad social universitaria. Las etapas que se ejecutaron para el desarrollo de este trabajo se describen a continuación: 
1. Diseño: se realizó una revisión sistemática de la bibliografía sobre los conceptos de responsabilidad social universitaria y de transferencia tecnológica, a fin de identificar prácticas de ejecución e identificar puntos de inflexión. Se consideraron estudios de revisión sobre estos temas.

2. Estrategia de búsqueda: las búsquedas se realizaron en las bases de datos ISI y SCOPUS con los siguientes términos de búsqueda: "prácticas de responsabilidad social universitaria"; "mecanismos de transferencia"; "tecnología + responsabilidad social universitaria"; "responsabilidad social + transferencia tecnológica".

3. Criterios de inclusión y exclusión: se tuvieron en cuenta trabajos que insistieran en prácticas de responsabilidad social universitaria y aquellos que incluyeran aspectos sociales de la transferencia tecnológica.

4. Extracción de datos: a partir de la aplicación de los criterios de selección se eligieron 46 artículos como relevantes para el estudio.

5. Análisis de los datos: para el análisis de los datos se construyó una matriz de sistematización y recopilación de la información, se hizo una revisión comparativa de los textos y se extrajeron las ideas claves de los mismos que permitieran configurar los principales aportes en torno al tema.

\section{LA TRANSFERENCIA TECNOLÓGICA DESDE LA UNIVERSIDAD. ENFOQUE SOCIAL}

Hoy en día las universidades no son consideradas una torre de marfil, sino que han crecido hasta convertirse en un catalizador del crecimiento de las naciones más allá del desarrollo del capital humano, transformándose en la fuente más importante para la transferencia de tecnología, la investigación, el desarrollo y la innovación en la sociedad (Hamdan et al., 2011, p. 1284).

La investigación universitaria y las actividades relacionadas con la misma contribuyen de manera importante a las economías modernas, principalmente: (i) por el aumento de la productividad de la I + D aplicada en la industria, (ii) por el desarrollo de nuevos conocimientos y aportes técnicos, (iii) por la provisión de capital humano de gran valor, (iv) por el desarrollo de equipos y tecnología y ( $v$ ) por el diseño de conceptos y prototipos de nuevos productos y procesos que pueden tener algunos impactos sociales y económicos grandes e inesperados (Roessner et al., 2013, p. 23). En el marco de estos procesos las universidades deben gestionar las más amplias consecuencias sociales y medioambientales de sus acciones, más allá de los requisitos legales y normativos con los que operan (Mendez, 2011, p. 2).

Las universidades pueden alcanzar un comportamiento socialmente responsable y contribuir a un mejor posicionamiento de su gestión tecnológica a través del manejo de la cantidad y calidad de una investigación que refuerce la relación entre universidad y entorno social, y a través del desarrollo de programas de transferencia tecnológica capaces de impulsar la vinculación de la universidad con su entorno. Sin embargo, para ello es indispensable revisar la normativa universitaria, plantear políticas de trasferencia tecnológica realmente adaptadas a la realidad y especializar al personal en torno al tema (Martínez de Carrasquero, 2012, p. 364).

En este sentido las universidades, a consecuencia de su relación con las ciudades, pueden convertirse en "buenos o malos vecinos" (O'Mara, 2012, citado en Gaete Quezada, 2015, p. 277) en relación con el papel fundamental que los procesos de transferencia tecnológica juegan en el desarrollo económico de las comunidades locales. Como ejemplo, una práctica de responsabilidad social que en el marco de la transferencia de tecnología tiene un impacto relevante es la de asesorías a las comunidades en diversos ámbitos (Martínez, Mavarez, Rojas, Rodríguez y Carvallo, 2006, p. 2).

Analizando los conceptos asociados a la transferencia tecnológica y la forma a través de la cual estos procesos contribuyen a la responsabilidad social universitaria, se presentan algunos aspectos a partir de los cuales se podría considerar la transferencia tecnológica como una práctica de responsabilidad social, partiendo de las siguientes premisas:

i. La tercera misión de la universidad, en la que se enmarcan los procesos de transferencia tecnológica, está asociada a su contribución al desarrollo económico y social.

ii. El desarrollo de procesos de transferencia tecnológica, mediante el mecanismo que se aplique, implica transformaciones sociales que pueden impactar positiva o negativamente a los contextos donde se implementen.

iii. La formación de capital humano en sus diferentes niveles es una práctica de transferencia tecnológica que se puede ejecutar de manera responsable, desde los ámbitos de la enseñanza tradicional, como en las actividades de educación continuada o educación para el trabajo. 
iv. Los procesos de transferencia tecnológica tienen una repercusión directa con las áreas de influencia de los receptores de la tecnología.

v. La triada universidad-empresa-estado en el marco de los procesos de transferencia tecnológica favorece su impacto y las implicaciones sociales de estos procesos.

vi. El cambio en los modelos de gobernanza hacia una universidad más comprometida con la sociedad y sus partes interesadas.

La consideración de estos factores favorece el ejercicio de la transferencia tecnológica desde la perspectiva de la responsabilidad social, por lo que la universidad debe tomar conciencia e identificar acciones explícitas que permitan incorporar el carácter social desde esta práctica. En suma, a partir de la revisión de la bibliografía se identificaron aspectos inherentes a la transferencia tecnológica que también son propios del ejercicio social, los cuales se detallan a continuación y constituyen un referente para entender cómo la transferencia tecnológica puede ser considerada una práctica de responsabilidad social universitaria.

\section{El impacto social de la transferencia tecnológica}

Considerando el trabajo de sociólogos y antropólogos, Bozeman (2000, p. 630) destacó que la transferencia de tecnología se da en un contexto de cambio cultural, además de que existen formas en que la tecnología afecta al cambio, por lo que el interés en la evaluación de los resultados e impactos de los procesos de transferencia tecnológica va en aumento. Es así como actualmente, debido al creciente interés por la transferencia de tecnología orientada al valor social y público, las instituciones del gobierno incluyen en sus casos de éxito los impactos sociales de sus actividades de transferencia de tecnología y demuestran cómo la política pública no se centra solo en el enriquecimiento de los socios de transferencia de tecnología, sino más bien sobre los impactos sociales y económicos más amplios (Bozeman, Rimes y Youtie, 2015, p. 35).

Aun así estos cambios no han sido asumidos del todo, de tal forma que los datos sobre patentes y licencias se utilizan a menudo como indicadores para evaluar los esfuerzos de transferencia de tecnología de las universidades (Okamuro y Nishimura, 2009, p. 7), dejando de lado los resultados asociados a los beneficios e impactos a la sociedad. Sin embargo, estos resultados pueden tener una mirada en la que se destaque el aporte social de los mismos. Tal es el caso de las spin-off, que de acuerdo con Kanter (1999) son nuevos modelos empresariales que surgen de la investigación académica y agregan aspectos basados en conocimiento e innovación sostenible como resultado del proceso de cooperación entre las instituciones de investigación y las instituciones comerciales.

Lo anterior pone sobre la mesa la importancia de considerar los impactos que los productos o resultados de la transferencia de tecnología pueden generar en la sociedad y el hecho de que la relevancia social de la investigación desarrollada por la universidad es el principal objetivo de transferencia tecnológica, cobrando más importancia que la obtención de beneficios económicos.

\section{El cambio en el contrato ciencia-sociedad}

Gibbons (1999) argumentó que el contrato entre la ciencia y la sociedad se creó para sostener la producción de conocimiento fidedigno y que, al establecerse un nuevo contrato, se debe hacer de tal forma que asegure la producción de conocimiento socialmente robusto. Por lo que un nuevo contrato requiere la producción conjunta de conocimiento entre la sociedad y la ciencia, lo que implica un proceso en el cual tendrá que ser legitimado una y otra vez (Gibbons, 1999, citado en Mendez, 2012, p. 12). Este cambio en el contrato social para la ciencia representa la expectativa de que, a cambio de inversiones del gobierno, las universidades producirán una buena investigación pública que sirva al interés de la nación y resuelva sus problemas sociales (Mendez, 2012, p. 12).

Adicionalmente, Leydesdorff y Meyer (2010, p. 355) afirman que un nuevo contrato social se ha formado entre la academia, la industria y los gobiernos como principales socios en la producción de conocimiento, y la tercera misión ha permanecido latente, incluyendo nuevas formas de educación y compromisos a largo plazo con los valores sociales.

Frente a lo antes expuesto se hace explícito el desafío de la universidad, la cual no debe estar distante de los grandes problemas a los que se enfrenta el mundo hoy en día -los desafíos ambientales, las injusticias sociales, los conflictos armados, la intolerancia, la falta de respeto de los derechos humanos...- y debe tener un papel activo de participación en los espacios locales y globales para fomentar y apoyar una de manera activa una sociedad justa y sostenible (Boni y Gasper, 2012 , p. 6). Con base en este reto, las relaciones de la universidad con la industria pueden aparecer como "socialmente responsables" en la medida en que los beneficios se inviertan en la solución de los problemas de la sociedad (Ankrah y AL-Tabbaa, 2015, p. 400). 


\section{La divulgación de los resultados de la transferencia tecnológica}

Closs et al. (2012, p. 114) señalan que la publicación autorizada de una invención académica permite beneficiar a la sociedad; además puede ayudar a impulsar la imagen de la universidad y añadirle valor a la institución. Adicionalmente, la difusión de experiencias empresariales de éxito ayuda a cambiar la expectativa personal o la imagen social y constituye un jalonador de las mismas. Es el caso de las universidades que promueven sus actividades de colaboración con la participación de empresarios externos e internos pueden constituirse en referente para futuros desarrollos. (Guerrero et al., 2014, p. 423).

En este contexto, cabe citar la denominada cuarta misión (la promoción) de la universidad con relación a la transferencia de tecnología, concebida como un vehículo de relaciones públicas inteligente que permite a las universidades diferenciar el "papel único" de la oficina de transferencia de tecnología de sus otras misiones principales (Bueno Campos y Casani Fernández de Navarrete, 2007; Hayter, 2016).

\section{El capital social}

El capital social lo constituyen los activos de propiedad colectiva que residen y derivan de las relaciones estables entre los actores y las unidades sociales, es la buena voluntad a disposición de la comunidad universitaria (estudiantes, empresarial, académicos), y su origen está en la estructura y en el contexto de las relaciones del actor (de mercado, sociales o jerárquicas). Por lo tanto, su efecto se deriva de la información, la influencia y la solidaridad que la universidad coloca a disposición de los actores con los que se relaciona (Adler y Kwon, 2002, p. 23). El capital social constituye un importante motor de la eficacia de las relaciones de la universidad con los diferentes actores involucrados (Al-tabbaa y Ankrah, 2016, p. 2).

El capital social es concebido también como un activo donde la confianza está basada en las relaciones sociales y en las interacciones continuas; así mismo abarca el valor colectivo de la relación y la reciprocidad y otros enlaces que conectan redes de individuos e instituciones, constituyendo un capital de ayudas sociales, coordinación y cooperación para beneficio mutuo (Wang y Shapira, 2012, p. 202); se relaciona directamente con la confianza entre los actores que se relacionan, y juega un papel determinante en la calidad y efectividad de los resultados de las relaciones.
Para el caso de los científicos universitarios su capital social se desarrolla a partir de sus redes de investigación y de otras relaciones científicas, además de que se complementa con el denominado capital posicional, el cual se asocia con el prestigio y la reputación de las instituciones a las que están vinculados (Wang y Shapira, 2012, p. 198). A lo anterior se suma que la estructura social de la economía regional y el tejido de las relaciones sociales en el que se incluye una universidad influye fuertemente en la densidad de los contactos que unen a los científicos con las personas en la industria, y además afecta a la densidad de las redes a través de las cuales se puede comercializar el conocimiento universitario (Casper, 2013, p. 1314; Hamdan et al., 2011, p. 1286).

\section{El trabajo articulado con las partes interesadas}

Con relación al establecimiento de redes con las partes interesadas, cada vez es más marcada la incorporación de las universidades en la generación de conocimiento y transferencia de tecnología con mayor pertinencia social y vinculación con las comunidades, a partir de la unión de esfuerzos de todas las disciplinas (Hamdan et al., 2011, p. 1286).

Para alcanzar mayores niveles de eficiencia en los resultados propuestos se necesita establecer redes de cooperación entre los principales actores vinculados. Teniendo en cuenta que el desarrollo tecnológico no solo depende de las universidades, el proceso ha trascendido hacia todos los sectores, para el logro del progreso social y tecnológico del país. Por lo que la participación de la comunidad, del empresario y del gobierno, convertidos en aliados estratégicos, operativos y estructurales, conjugan y coadyuvan a la ejecución de proyectos tecnológicos con pertinencia, utilidad y viabilidad en el contexto en que se desarrollan (Sira y Pérez, 2015, p. 959).

Particularmente, las políticas de desarrollo regional en la mayoría de los países de América Latina, como en muchas economías en desarrollo, se ha orientado hacia la promoción de la colaboración de las partes interesadas en la investigación y difusión de la tecnología (Theodorakopoulos, Bennett y Sánchez Preciado, 2014, p. 647). Esto ha promovido una coalición de partes interesadas en los procesos de transferencia de tecnología, que se apoya en una política equilibrada y en mecanismos tales como agendas, planes, sistemas para la evaluación y en tecnologías y espacios de intercambio de conocimiento como foros, grupos directivos, eventos de capacitación y visitas de asistencia (Theodorakopoulos et al., 2014, p. 656). 
Finalmente cabe resaltar que, en términos de expectativas, los diferentes grupos de interés de la universidad representan principalmente un instrumento de legitimidad que puede favorecer el proceso de aceptación y aprobación de sus actividades en su entorno social, aunque actualmente desempeñan un papel menor entre las instituciones, a pesar de la gran influencia que tienen en cualquier organización (Garde Sánchez et al., 2013, p. 552).

\section{Una universidad comprometida}

De acuerdo con lo expresado por Conway et al. (2009, p. 11) ha habido un cambio asociado a la naturaleza de la producción de conocimiento que ha llevado desde la transferencia de conocimiento a la coproducción; y además el concepto de transferencia de tecnología basado en la idea de que una sola oficina podría atender las solicitudes de gestión de las empresas, identificar qué universidad podría resolver esos problemas y establecer un contrato para que la universidad proveyera una solución no ha logrado aprovechar todo el potencial de las partes involucradas, debido a que la naturaleza de la innovación ha cambiado para volverse más interactiva (Conway et al., 2009, p. 23).

Una tendencia global emergente es promover el compromiso social. Algunos autores del Reino Unido afirman que las universidades desempeñan un papel clave no solo en el desarrollo económico sino también en el social, especialmente porque están vinculadas al desarrollo regional (Benneworth, Boer y Jongbloed, 2015; European Union, 2011). Este concepto generalmente se relaciona con lazos de beneficio mutuo a nivel local, entre los miembros de la universidad y las comunidades dentro de la ciudad y región de origen. Esta es una dimensión más sobresaliente en América Latina (Mora, Aguiar Serra y Vieira, 2017, p. 6). Particularmente Goddard (2009), a través del concepto de universidad cívica destaca la importancia de que la universidad integre la docencia y la investigación al servicio de agendas académicas y sociales/industriales, y además destaca que el compromiso activo de las universidades con las regiones está en su contribución al sistema regional de innovación mediante la generación de capacidades.

Esto incluye la contribución de la educación superior al desarrollo social, cultural y ambiental, mediante la participación formal e informal y la representación externa como actor institucional en las redes regionales de aprendizaje, innovación y gobernabilidad (Uyarra, 2010 , p. 1238). Estos cambios han creado nuevas pre- siones para las universidades, particularmente sobre sus modelos de gobernanza, y han llevado desde la toma de decisiones dirigida por los expertos académicos hacia la toma de decisiones dirigida por los consumidores (Conway et al., 2009, p. 25).

\section{LA TRANSFERENCIA TECNOLÓGICA COMO PRÁC- TICA DE RESPONSABILIDAD SOCIAL UNIVERSITARIA}

Como se ha presentado en este trabajo, en el alcance de la transferencia tecnológica se pueden considerar implicaciones que contribuyen a su enfoque social y se deben incorporar acciones explícitas que refuercen y hagan operativo el cumplimiento de los compromisos con el entorno.

Estas acciones permitirían enriquecer los procesos de transferencia tecnológica y de igual forma constituyen una base sólida para que las universidades sustenten una gestión socialmente responsable. Las siguientes proposiciones colocan en su contexto la transferencia tecnológica frente a los desafíos de la responsabilidad social universitaria.

Proposición 1: Incorporar problemáticas y necesidades sociales en los procesos de transferencia tecnológica para la generación de transformaciones e impactos sociales, económicos y culturales en la sociedad. Desde esta perspectiva se propone que los procesos de transferencia tecnológica no pueden estar a espaldas de la realidad social en la que está inmersa la universidad y de los actores del entorno en que se ubica. En este sentido los proyectos realizados deben contemplar variables como la sostenibilidad y la gestión ambiental, tanto desde el diseño como en la ejecución y deben hacer un adecuado uso de los indicadores de impacto, más allá de los de resultados. La universidad debe iniciar un seguimiento de los proyectos posterior a su finalización y llevar los registros requeridos para hacer verificar los efectos de su implementación. A fin de profundizar en lo anterior se deben promover investigaciones empíricas que identifiquen casos de procesos de transferencia tecnológica que incorporen necesidades y problemáticas explicitas de comunidades asociadas a la universidad. Es de destacar que esta proposición se articuló con lo propuesto en la proposición 4, lo que implica que para el logro de estos procesos de transferencia con un mayor impacto social se requiere contar con una participación activa de las partes involucradas, no desarrollar proyectos aislados, sino que por el contrario vinculen a actores relacionados con la universidad. 
Proposición 2: Vincular el compromiso social en las políticas institucionales como eje del proceso de responsabilidad social universitaria y como promotor en procesos institucionales tales como la transferencia tecnológica, en el contexto de un nuevo contrato social de conocimiento socialmente robusto. El compromiso de la responsabilidad social de la transferencia tecnológica no puede ser un proceso improvisado, sino que debe responder y estar articulado con relación a los planes institucionales, desde la dirección institucional hasta los planes del área de investigación y los grupos de investigación y de igual forma en la misión y visión de estos procesos. Por lo que esta proposición lleva a estudiar las condiciones organizativas de la universidad que lleven a procesos de transferencia tecnológica con compromiso social, de tal forma que permitan estudiar cómo este proceso es una acción no aleatoria sino deliberada en estas instituciones.

Proposición 3: Divulgar los procesos y resultados de transferencia tecnológica como insumos para potenciales procesos de transformación social, favoreciendo procesos de apropiación en la comunidad. Esto evidencia cómo la apropiación social de los resultados de la transferencia tecnológica contribuye a la responsabilidad social universitaria. La universidad debe ser capaz de llevar su lenguaje al lenguaje de la comunidad y al de los actores relacionados, informando sobre los resultados del conocimiento generado y sobre cómo esos resultados pueden ser utilizados para su beneficio y para el desarrollo futuro; esto en condiciones adecuadas para su comprensión, de tal forma que favorezca investigaciones y trabajos futuros. Los procesos de divulgación no pueden ser confundidos con la promoción y comercialización de lo que hace la universidad, pero si pueden ser complementados por los mismos, deben ser entendidos como una forma en que la universidad se encarga de que su trabajo realmente llegue a quienes podrían finalmente beneficiarse del mismo y que la sociedad conozca cómo la universidad trabaja para la mejora de sus necesidades y problemas. Este aspecto abre la posibilidad de estudios sobre los medios y mecanismos de promoción y divulgación de los resultados de transferencia y cómo llegan a diferentes sectores asociados con la universidad.

Proposición 4: Considerar a los actores implicados como parte del capital social de la universidad e investigadores en el marco de los procesos de transferencia tecnológica. Este aspecto constituye un común denominador con las proposiciones anteriores, puesto que un trabajo articulado con las partes inte- resadas redunda significativamente sobre los efectos sociales de la transferencia, debe estar claro en los planes y políticas diseñados para ello y debe estar apoyado en mecanismos de comunicación y divulgación adecuados. En este sentido la universidad debe tener parámetros claros que aseguren un adecuado uso de su capital social no solo con la comunidad científica sino con otros actores involucrados para enriquecer sus procesos de transferencia. La universidad no puede encerrase en una cápsula, sino que debe estar abierta a un diálogo constante con las partes involucradas. Por ello trabajos futuros permitirán estudiar las redes y trabajos colaborativos con diferentes actores y socios en la universidad a fin de identificar los medios y estrategias de trabajo con cada uno y los proyectos y resultados que se obtengan de este tipo de trabajos.

\section{ESTRATEGIAS DE RESPONSABILIDAD SOCIAL EN LA TRANSFERENCIA TECNOLÓGICA}

Tomando como base el análisis de las características y prácticas de responsabilidad social universitaria y de la transferencia tecnológica, se identificaron ciertas estrategias que permitirían considerar la transferencia tecnológica desde la perspectiva de la responsabilidad social universitaria y que favorecerían la generación de indicadores que hagan evidente esta gestión y la imagen de la universidad en su entorno social, como se presenta la tabla 1.

Estas estrategias constituyen un referente para que las universidades puedan incorporar de manera formal los procesos de transferencia tecnológica como resultado de su gestión en responsabilidad social y así mejorar su impacto en el entorno.

\section{DISCUSIÓN Y CONCLUSIONES}

La bibliografía ha reconocido tradicionalmente que los procesos de transferencia tecnológica se asocian principalmente con relaciones directamente establecidas con las empresas, entendiéndolos como los principales demandantes de tecnología. Como resultado de este tipo de vinculaciones se generan productos de conocimiento tales como patentes, spin-off, licencias y resultados de consultorías especializadas y se favorece la producción científica de los investigadores y la innovación en las empresas. En esta bibliografía poco reconocimiento se da a la vinculación con otros actores sociales y a los impactos que estos procesos de transferencia generan a los problemas del contexto social. 
Tabla 1. Estrategias para la responsabilidad social de la transferencia tecnológica

\begin{tabular}{|c|c|}
\hline Características de la RSU & Características de la TT desde el enfoque social \\
\hline $\begin{array}{l}\text { 1. Valores y principios institucionales. } \\
\text { 2. Currículo oculto. } \\
\text { 3. Clima organizativo. } \\
\text { 4. Buena vecindad. } \\
\text { 5. Comunicación. } \\
\text { 6. Involucrar a las partes interesadas. } \\
\text { 7. Enfoque hacia el desarrollo sostenible. }\end{array}$ & $\begin{array}{l}\text { 1. Transferencia tecnológica con valor social. } \\
\text { 2. Indicadores de impacto social. } \\
\text { 3. La sostenibilidad económica, social y ambiental de la TT. } \\
\text { 4. Conocimiento socialmente robusto. } \\
\text { 5. Divulgación de la investigación y apropiación social del } \\
\text { conocimiento. } \\
\text { 6. Capital y cohesión social. } \\
\text { 7. Universidad comprometida. } \\
\text { 8. Relación con los actores implicados. }\end{array}$ \\
\hline
\end{tabular}

1. Desarrollar políticas institucionales que promuevan el compromiso e impacto social de la TT.

2. Promover el desarrollo de relaciones mutuamente beneficiosas con la comunidad circunvecina a la Universidad.

3. Incluir indicadores de impacto social en el proceso de la TT.

4. Implementar acciones de sostenibilidad social y ambiental en los proyectos de TT.

5. Incorporar a la sociedad y sus problemas sociales en los procesos de TT.

6. Diseñar e implementar políticas y planes de divulgación y comunicación de la TT.

7. Desarrollar programas de apropiación social del conocimiento en comunidades vinculadas a la Universidad.

8. Establecer relaciones continuas y estructurales con las partes interesadas.

9. Desarrollar capacidades institucionales de cohesión social y de trabajo en red con sectores estratégicos para la universidad.

10. Establecer redes y alianzas con las partes interesadas en procesos de TT.

Fuente: Elaboración propia.

En estos últimos años se ha reconocido el compromiso que las universidades tienen no solo con el desarrollo tecnológico y económico sino social, por lo que se están realizando cada vez más esfuerzos para crear condiciones institucionales que respalden este tipo de acciones. En línea con esto está lo establecido en el marco del concepto de responsabilidad social universitaria, el cual establece lineamientos y condiciones de trabajo que contribuyen a fortalecer el vínculo de la universidad con sus partes interesadas y con las necesidades de su contexto de influencia.

La responsabilidad social plantea a las universidades el desafío de contribuir al desarrollo social y a la sostenibilidad ambiental, de acuerdo con sus misiones y atendiendo a las necesidades y a la problemática de los grupos de interés con los que interactúan. En relación con esto la transferencia tecnológica desde la universidad constituye una oportunidad para contribuir a los procesos de crecimiento económico, social y cultural, apoyados en las capacidades científico-tecnológicas de la universidad, que llevan a relaciones cercanas con la sociedad y con sectores estratégicos en sus áreas de influencia. Esta última afirmación pone de relieve las relaciones existentes entre ambos conceptos y cómo uno puede llegar a sostener al otro, lo que hace evidente la posibilidad de desarrollar la transferencia tecnológica como una práctica de responsabilidad social.
Particularmente en el campo de la transferencia tecnológica diferentes estudios han destacado como un factor preponderante en el enfoque social de la transferencia tecnológica la vinculación de las partes interesadas (Martínez et al., 2006; Theodorakopoulos et al., 2014) y usuarios de estos procesos (Greiner y Franza, 2003 , p. 170). Además se reconocen aspectos operativos de estos procesos, de tal forma que trabajos como el de Sira y Pérez (2015, p. 973) presentan un modelo operativo que identifica los procesos de transferencia tecnológica enmarcados en la responsabilidad social, poniendo el énfasis en factores sociales, culturales y humanos que inciden en estos procesos e insistiendo en las relaciones con actores externos a la universidad; y Martínez de Carrasquero (2012, p. 527), por su parte, indicó que los canales de transferencia tecnológica que han logrado ser transferidos al entorno social con mayor éxito son la asistencia técnica, las asesorías, los cursos de capacitación y la consultoría.

Frente a lo antes expuesto, los resultados de este trabajo ratifican que la transferencia tecnológica puede ser considerada como una práctica social que no solo se lleva a cabo involucrando a las partes interesadas, sino también dando respuesta a las demandas del entorno, así como cumpliendo procesos adecuados de divulgación. Esto debe estar establecido en la política institucional de tal forma que se asegure con- 
tar con las condiciones y el respaldo de las instituciones. Este trabajo propone unas líneas de acción que pueden ser referenciadas por las universidades en el marco de su proceso institucional de responsabilidad social universitaria, así como en el diseño de los indicadores del mismo.

En este sentido este trabajo arrojó cuatro proposiciones que constituyen otras tantas oportunidades para futuras investigaciones y que representan coyunturas explícitas para sustentar cómo los procesos de transferencia tecnológica llevan a una responsabilidad social ejercida desde la universidad.

Como conclusión general se puede afirmar que la revisión bibliográfica permitió identificar elementos conceptuales y operativos de la transferencia tecno- lógica que permiten sustentar procesos de responsabilidad social llevados a cabo desde la universidad. Este trabajo presenta como principal aporte la identificación explícita de estas prácticas que permitirían a la universidad identificar y hacer visible la manera en que su trabajo de transferencia también contribuye a su compromiso socialmente responsable.

Este trabajo puede ser un punto de referencia para el desarrollo de futuras investigaciones a fin de realizar estudios de caso e identificar las prácticas implementadas en las universidades, tanto de países desarrollados como de Latinoamérica, y los mecanismos que han desarrollado frente al reto de incorporar los procesos de responsabilidad social universitaria e identificar constructos teóricos a partir de estos referentes.

\section{BIBLIOGRAFÍA}

Adler, P. S. y Kwon, S.-W. (2002). Social Capital: Prospects for a New Concept. The Academy of Management Review, 27 (1), pp. 17-40. Disponible en: http://www.jstor.org/stable/4134367

Al-Tabbaa, O. y Ankrah, S. (2016). Technological Forecasting \& Social Change Social capital to facilitate ' engineered ' university - industry collaboration for technology transfer : A dynamic perspective. Technological Forecasting \& Social Change, 104, pp. 1-15. https://doi.org/10.1016/j. techfore.2015.11.027

Ankrah, S. y AL-Tabbaa, O. (2015). Universities-industry collaboration: A systematic review. Scandinavian Journal of Management, 31 (3), pp. 387-408. https://doi.org/10.1016/j. scaman.2015.02.003

Araque Acevedo, J., Rubio Guerrero, G. y Uribe Macías, M. (2015). Responsabilidad social universitaria: teoría y aplicación. Caso de análisis: Universidad del Tolima.

Aristimuño, M. y Rodriguez Monroy, C. (2014). Responsabilidad social universitaria. Su gestión desde la perspectiva de directivos y docentes. Estudio de caso: una pequeña universidad latinoamericana. Interciencia. Revista de Ciencia y Tecnología de América, 39 (6), pp. 375-383.

Barnes, T., Pashby, I. y Gibbons, A. (2002). Effective university-Industry interaction: A multi-case evaluation of collaborative R\&D projects. European
Management Journal, 20 (3), pp. 272-285. https://doi.org/10.1016/ S0263-2373(02)00044-0

Benneworth, P., Boer, H. de y Jongbloed, B. (2015). Between good intentions and urgent stakeholder pressures: institutionalizing the universities' third mission in the Swedish context. European Journal of Higher Education, 5 (3), pp. 280-296. https://doi.or g/10.1080/21568235.2015.1044549

Beraza Garmendia, J. M. y Rodríguez Castellanos, A. R. (2007). La evolución de la misión de la universidad. Revista de Dirección y Administración de Empresas, 14, pp. 25-56. Disponible en: https://addi.ehu.es/bitstream/ handle/10810/9908/Revista14_02. pdf?sequence $=1$

Bierly III, P. E., Damanpour, F. y Santoro, M. D. (2009). The application of external knowledge: Organizational conditions for exploration and exploitation. Journal of Management Studies, 46 (3), pp. 481-509. https://doi.org/10.1111/j.14676486.2009.00829.x

Boni, A. y Gasper, D. (2012). Rethinking the Quality of Universities: How Can Human Development Thinking Contribute? Journal of Human Development and Capabilities, 13 (3), pp. 451-470. https://doi.org/10.1080/19 452829.2012.679647

Boyle, M. E. (2007). Learning to neighbor? Service-learning in context. Journal of Academic Ethics, 5 (1), pp. 85-104. https://doi.org/10.1007/ s10805-007-9045-5

Bozeman, B. (2000). Technology transfer and public policy: a review of research and theory. Research Policy, 29 (4-5), pp. 627-655. https://doi.org/10.1016/ S0048-7333(99)00093-1

Bozeman, B., Rimes, H. y Youtie, J. (2015). The evolving state-of-the-art in technology transfer research : Revisiting the contingent effectiveness model. Research Policy, 44 (1), pp. 34-49. https://doi.org/10.1016/j.respol.2014.06.008

Bueno Campos, E. y Casani Fernández de Navarrete, F. (2007). La tercera misión de la Universidad: enfoques e indicadores básicos para su evaluación. Economía Industrial, 366, pp. 43-60. Disponible en: http:// europa.sim.ucm.es/compludoc/ $A A$ ?articulold $=580582$

Cardona Zuleta, E. (2010). Estrategias para la materialización de las políticas de resposabilidad social en el marco de los programas regionalizados. Estudios de Derecho, 67 (149), pp. 165-188.

Casper, S. (2013). The spill-over theory reversed: The impact of regional economies on the commercialization of university science. Research Policy, 42 (8), pp. 1313-1324. https://doi. org/10.1016/j.respol.2013.04.005

Chiappetta Jabbour, C. J. (2010). Greening of business schools: a systemic view. International Journal 
of Sustainability in Higher Education, 11 (1), pp. 49-60. https://doi. org/10.1108/14676371011010048

Closs, L., Ferreira, G. C., Soria, A. F., Sampaio, C. H. y Perin, M. (2012). Organizational factors that affect the university-industry technology transfer processes of a private university. Journal of Technology Management and Innovation, 7 (1), pp. 104-117. https://doi.org/10.4067/S071827242012000100007

Conway, C., Humphrey, L., Benneworth, P., Charles, D. y Younger, P. (2009). Characterising modes of university engagement with wider society. A literature review and survey of best practice. [En línea]. Disponible en: https://strathprints.strath. ac.uk/48210/

D'Este, P. y Perkmann, M. (2011). Why do academics engage with industry? The entrepreneurial university and individual motivations. The Journal of Technology Transfer, 36 (3), pp. 316-339. https://doi.org/10.1007/ s10961-010-9153-z

Esfijani, A., Hussain, F. y Chang, E. (2013). University social responsibility ontology. International Journal of Engineering Intelligent Systems, 21, (4), pp. 271-281.

Etzkowitz, H. y Leydesdorff, L. (2000). The dynamics of innovation: from National Systems and "Mode 2" to a Triple Helix of university-industry-government relations. Research Policy, 29 (2), pp. 109-123. https://doi.org/10.1016/ S0048-7333(99)00055-4

Freitas, S. de, Mayer, I., Arnab, S. y Marshall, I. (2014). Industrial and academic collaboration: hybrid models for research and innovation diffusion. Journal of Higher Education Policy and Management, 36 (1), pp. 2-14. https://doi.org/10.1080/136008 oX.2013.825413

Fromhold-Eisebith, M. y Werker, C. (2013). Universities' functions in knowledge transfer: A geographical perspective. The Annals of Regional Science, 51 (3), pp. 621-643. https:// doi.org/10.1007/s00168-013-0559-z

Gaete, R. (2011). La responsabilidad social universitaria como desafío para la gestión estratégica de la Educación Superior: el caso de España. Revista de Educacion, 355, pp. 109-133.
Gaete Quezada, R. A. (2015). El concepto de responsabilidad social universitaria desde la perspectiva de la alta dirección. Cuadernos de Administración, 31 (53), pp. 97-107. https://doi. org/10.25100/cdea.v31i53.20

Garde Sánchez, R., Rodríguez Bolívar, M. P. y López-Hernández, A. M. (2013). Online disclosure of university social responsibility: a comparative study of public and private US universities. Environmental Education Research, 19 (6), pp. 709-746. https://doi.org/10.1 080/13504622.2012.749976

Gasca-Pliego, E. y Olvera-García, J. C. (2011). Construir ciudadanía desde las universidades, responsabilidad social universitaria y desafíos ante el siglo XXI. Convergencia, 18 (56), pp. 37-58.

Gibbons, M. (1999). Science's new social contract with society. Nature, 402 (6761 supp), C81-C84. https://doi. org/10.1038/35011576

Gibbons, M., Limoges, C., Nowotny, H., Schwartzman, S., Scott, P. y Trow M. (1994). The new production of knowledge: The dynamics of science and research in contemporary societies. London: Sage.

Goddard, J. (2009). Reinventing the civic university. National Endowment for Science, Technology and the Arts (NESTA). [En línea]. Disponible en: https://www.ncl.ac.uk/media/wwwnclacuk/curds/files/Reinventing\%20 the\%20civic\%20university.pdf

Greiner, M. A. y Franza, R. M. (2003). Barriers and Bridges for Successful Environmental Technology Transfer. Journal of Technology Transfer, 28 (2), pp. 167-177. https://doi. org/10.1023/A:1022998617118

Guadrón, L., Silva, M., Acosta, Y. y Piñero, L. (2012). La docencia en el marco de la responsabilidad social universitaria. Opcion, 28 (68), pp. 257-272. Disponible en: https://goo.gl/UxQqsZ

Guerrero, M., Urbano, D., Cunningham, J. y Organ, D. (2014). Entrepreneurial universities in two European regions: A case study comparison. The Journal of Technology Transfer, 39 (3), pp. 415-434. https://doi.org/10.1007/ s10961-012-9287-2

Hamdan, H., Yusof, F., Omar, D., AbduIlah, F., Nasrudin, N. y Abullah, I. C. (2011). University Industrial Linkages : Relationship Towards Economic
Growth and Development in Malaysia. International Journal of Social, Behavioral, Educational, Economic, Business and Industrial Engineering, 5 (10), pp. 1284-1291.

Hayter, C. S. (2016). A Social Responsibility View of the "Patent-Centric Linear Model" of University Technology Transfer. Duquesne Law Review, 54, pp. 7-52.

Kanter, R. M. (1999). From spare change to real change. The social sector as beta site for business innovation. Harvard Business Review, 77 (3), pp. 122-132.

Larrán Jorge, M. y Andrades Peña, F. J. (2014). Determinants of corporate social responsibility and business ethics education in Spanish universities. Business Ethics: A European Review, 23 (2), pp. 139-153. https://doi. org/10.1111/beer.12041

Leydesdorff, L. y Etzkowitz, H. (1996). Emergence of a Triple Helix of university-industry-government relations. Science and Public Policy, 23 (5), pp. 279-286.

Leydesdorff, L. y Meyer, M. (2010). The decline of university patenting and the end of the Bayh-Dole effect. Scientometrics, 83 (2), pp. 355-362. https://doi.org/10.1007/s11192-0090001-6

Martin, B. R. (2003). The changing social contract for science and the evolution of the university. En Geuna, A., Salter, A. J. y Steinmueller, W. E. (eds.) Science and Innovation: Rethinking the Rationales for Funding and Governance. Edward Elgar Publishing, pp. 7-29. https://doi. org/10.4337/9781781950241.00011

Martínez, C., Mavarez, R., Rojas, L., Rodríguez, J. y Carvallo, B. (2006). La responsabilidad social como instrumento para fortalecer la vinculación universidad-entorno social. I Congreso Iberoamericano de Ciencia, Tecnología, Sociedad e innovación CTS+l, México D.F., del 19 al 23 de junio de 2006.

Martínez de Carrasquero, C. (2012). Responsabilidad social universitariatransferencia tecnológica en su vinculación con el entorno social. Opción, 28 (68), pp. 512-528.

Mendez, M. (2011). University Social Responsibility: Balancing Economic 
\& Societal Benefits of University Research. The Journal of Science Policy \& Governance, 1 (1), pp. 1-25. Disponible en: https://issuu.com/jofspg/ docs/university_social_responsibility

Merchán Hernández, C. (2012). Las relaciones de las empresas con las universidades: estrategias y dinámicas del proceso de cooperación a nivel regional. Arbor, 188 (753), pp. 193-209. https://doi.org/10.3989/ arbor.2012.753n1012

Mora, J.-G., Aguiar Serra, M. y Vieira, M.J. (2017). Social Engagement in Latin American Universities. Higher Education Policy, 31 (4), pp. 513-534. https:// doi.org/10.1057/s41307-017-0069-1

O'Mara, M. P. (2012). Beyond town and gown: university economic engagement and the legacy of the urban crisis. The Journal of Technology Transfer, 37 (2), pp. 234-250. https://doi. org/10.1007/s10961-010-9185-4

Okamuro, H. y Nishimura, J. (2009). Impact of University Intellectual Property Policy on the Performance of University-Industry Research Collaboration. The Journal of Technology Transfer, 38 (3), pp. 273-301. https:// doi.org/10.1007/s10961-012-9253-z

Roessner, D., Bond, J., Okubo, S. y Planting, M. (2013). The economic impact of licensed commercialized inventions originating in university research. Research Policy, 42 (1), pp. 23-34. https://doi.org/10.1016/j.respol.2012.04.015

Siegel, D. S., Veugelers, R. y Wright, M. (2007). Technology transfer offices and commercialization of university intellectual property: Performance and policy implications. Oxford Review of Economic Policy, 23 (4), pp. 640-660. https://doi. org/10.1093/oxrep/grm036

Siegel, D. S., Waldman, D. A., Atwater, L. E. y Link, A. N. (2003). Commercial knowledge transfers from universities to firms: improving the effectiveness of university-industry collaboration. The Journal of High Technology Management Research, 14 (1), pp. 111133. https://doi.org/10.1016/S10478310(03)00007-5
Sira, S. y Pérez, R. (2015). Modelo operativo de transferencia tecnológica para promover la interacción universitaria con los sectores externos. Revista Digital de Investigación y Posgrado de la Universidad Nacional Experimental Politécnica "Antonio José de Sucre", Vicerrectorado Barquisimeto, 5 (4), pp. 958-976.

Siracusa, G., Ardiles, A. y Pulido, S. (2012). Gerencia universitaria postmoderna en el paradigma de la responsabilidad social. Revista Venezolana de Gerencia, 17 (58), pp. 307-320. Disponible en: http://www.redalyc. org/pdf/290/29023348007.pdf

Theodorakopoulos, N., Bennett, D. y Sánchez Preciado, D. J. (2014). Intermediation for technology diffusion and user innovation in a developing rural economy: a social learning perspective. Entrepreneurship \& Regional Development, 26 (7/8), pp. 645-662. https://doi.org/10.1080/08985626.2 014.971077

Torres Pernalete, M. y Trapága Ortega, M. (2010). Responsabilidad social de la universidad: retos y perspectivas. Paidós.

Tünnermann Bernheim, C. (2010). La reforma universitaria de Córdoba. Revista de Educación Superior y Sociedad, 9 (1), pp. 103-127.

Uyarra, E. (2010). Conceptualizing the Regional Roles of Universities, Implications and Contradictions. European Planning Studies, 18 (8), pp. 1227-1246. https://doi. org/10.1080/09654311003791275

Vallaeys, F. (2006). Breve marco teórico de responsabilidad social universitaria. Disponible en: https://www.econo.unlp.edu.ar/frontend/media/86/ 10986/10ec2d7e617a62eba0696821 196efeae.pdf

Vallaeys, F., Cruz, C. de la y Sasia, P. M. (2009). Responsabilidad Social Universitaria. Manual de primeros pasos. McGraw-Hill Interamericana Editores.

Vega Jurado, J., Manjarrés Henríquez, L., Castro Martínez, E. y Fernández de Lucio, I. (2011). Las relaciones universidad-empresa: tendencias y desafíos en el marco del Espacio Iberoameri- cano del Conocimiento. Revista lberoamericana de Educación, 4 (57), pp. 109-124.

Vera Salazar, P. H., Galvis-Lista, E. y González Zabala, M. P. (2013). Concretando la tercera misión $(3 \mathrm{~m})$ de la universidad pública regional. Impactos y percepciones de un proyecto de extensión. Caso Universidad del Magdalena. Revista Clío América, 7 (14), pp. 135-152.

Wang, Y., Hu, R., Li, W. y Pan, X. (2016). Does teaching benefit from universityindustry collaboration? Investigating the role of academic commercialization and engagement. Scientometrics, 106 (3), pp. 1037-1055. https://doi. org/10.1007/s11192-015-1818-9

Wang, J. y Shapira, P. (2012). Partnering with universities: A good choice for nanotechnology start-up firms? Small Business Economics, 38 (2), pp. 197215. https://doi.org/10.1007/s11187009-9248-9

\section{Otros recursos}

Conferencia Mundial sobre la Educación Superior. La educación superior en el siglo XXI. Visión y acción. UNESCO. París, 5-9 de octubre de 1998. [En línea]. Disponible en: http://unesdoc.unesco.org/ images/0011/001163/116345s.pdf

Conferencia Mundial sobre la Educación Superior - 2009. La nueva dinámica de la educación superior y la investigación para el cambio social y el desarrollo (Sede de la UNESCO, París, 5-8 de julio de 2009) 1-9. [En línea]. Disponible en: http://www.unesco. org/education/WCHE2009/comunicado_es.pdf

European Union (2011). Connecting Universities to Regional Growth: A Practical Guide. [En línea]. Disponible en: http://ec.europa.eu/regional_policy/ sources/docgener/presenta/universities2011/universities2011_en.pdf

Vallaeys, F. y Carrizo, L. (2006). Hacia la construcción de indicadores de responsabilidad social universitaria. [En línea]. Disponible en: https://durs.cayetano.edu.pe/images/Biblio/HerramientasRSU/HerramientasGestion/ indicadoresrsu.pdf 